\title{
Pressure and Stress Effects on Diffusion in Si
}

\section{Citation}

Aziz, Michael J., Pressure and Stress Effects on Diffusion in Si. Defect and Diffusion Forum, Vols. 153-155, pp 1-10. 1998. Trans Tech Publications.

\section{Published Version}

http://dx.doi.org/10.4028/www.scientific.net\%2FDDF.153-155.1

\section{Permanent link}

http://nrs.harvard.edu/urn-3:HUL.InstRepos:2797450

\section{Terms of Use}

This article was downloaded from Harvard University's DASH repository, and is made available under the terms and conditions applicable to Other Posted Material, as set forth at http:// nrs.harvard.edu/urn-3:HUL.InstRepos:dash.current.terms-of-use\#LAA

\section{Share Your Story}

The Harvard community has made this article openly available.

Please share how this access benefits you. Submit a story.

\section{Accessibility}


Special issue on "Ten Years of Diffusion in Silicon",

Defect and Diffusion Forum 153-155, 1-10 (1998).

\title{
Pressure and Stress Effects on Diffusion in Si
}

\author{
Michael J. Aziz* \\ Division of Engineering and Applied Sciences, Harvard University, Cambridge, MA 02138
}

Keywords: thermodynamics, diffusion mechanism, nonhydrostatic stress, biaxial stress, dopant diffusion, self diffusion, silicon, germanium, antimony, boron, arsenic.

\begin{abstract}
The thermodynamics of diffusion under hydrostatic pressure and nonhydrostatic stress is presented for single crystals free of extended defects. The thermodynamic relationships obtained permit the direct comparison of hydrostatic and biaxial stress experiments and of atomistic calculations under hydrostatic stress for any proposed mechanism. Atomistic calculations of the volume changes upon point defect formation and migration, and experiments on the effects of pressure and stress on the diffusivity, are reviewed. For $\mathrm{Sb}$ in $\mathrm{Si}$, using as input the results of $a b$ initio calculations of the effect of hydrostatic pressure on diffusion by the vacancy mechanism, the thermodynamic relationships successfully account for the measured effect of biaxial stress on diffusion with no free parameters. For other cases, missing parameters are enumerated and experimental and calculational procedures outlined.
\end{abstract}

\section{INTRODUCTION}

Because understanding and controlling diffusion related phenomena become increasingly important as semiconductor device dimensions decrease, diffusion in silicon has been heavily studied [1]. Despite this emphasis, substantial disagreements remain about the relative concentrations and mobilities of the point defects involved in the diffusion of many substitutional elements in Si. Such disagreements arise frequently because the interpretation of diffusion experiments in terms of atomistic mechanisms is often indirect and model-based [2].

A study of the dependence of the diffusivity, $D$, on pressure, $p$, and stress, $\sigma$, can provide valuable direct information to help elucidate the atomistic mechanism(s) of diffusion $[3,4]$. It can permit conclusions to be drawn about the predominant point defect mechanism independent of the assumptions inherent in the currently used kinetic models. The only necessary assumption is that the experimental time scale is either very long or very short compared to that for point defect equilibration between the experimental diffusion zone and the nearest surface. In what follows, we assume the former.

In addition, nonhydrostatic stresses in crystalline strained-layer epitaxial semiconductors can be very large. An understanding of their effects on diffusion and crystallization is therefore an important part of the study of the stability of these materials. Although many are studying the effects of these stresses on the energetics of growth and other kinetic processes in strained epitaxial films, our emphasis has been on the study of the effects of stress on the point defect concentrations and mobilities.

The atomic diffusivity in a material with cubic crystallography is 


$$
D=\frac{1}{6} \lambda^{2} \Gamma P_{\mathrm{m}} f
$$

where $\lambda$ is the jump distance, $P_{\mathrm{m}}$ is the fraction of the atoms that are mobile, $\Gamma$ is the average jump rate of the mobile species, and departures from a true random-walk are accounted for by the correlation factor, $f$. For example, for diffusion of an impurity "A" by a normal vacancy mechanism, the mobile species (which is called an A-V complex) is A on a lattice site adjacent to a vacancy. $P_{m}$ and $\Gamma$ are given, respectively, by

$$
\begin{aligned}
& P_{\mathrm{m}}=\exp \left(-G_{f}^{\mathrm{o}} / k_{\mathrm{B}} T\right), \\
& \Gamma=v \exp \left(-G^{m} / k_{\mathrm{B}} T\right),
\end{aligned}
$$

where $G_{\mathrm{f}}^{\mathrm{o}}$ is the standard free energy of formation of the mobile species, the free energy of migration $G^{m}$ is the additional change in free energy when the species moves to the saddle point of its migration path, and $v$ is an effective vibrational frequency [5]. Substituting into Eq. (1), we obtain the familiar form for $D$ :

$$
D=\frac{1}{6} \lambda^{2} \vee f \exp \left(-\frac{G_{f}^{\mathrm{o}}+G^{m}}{k_{\mathrm{B}} T}\right)
$$

\section{DIFFUSION UNDER HYDROSTATIC PRESSURE}

The effect of pressure on $D$ is characterized by the activation volume, $V^{*}$ :

$$
V^{*} \equiv-k_{\mathrm{B}} T\left(\frac{\partial \ln D}{\partial p}\right)_{\mathrm{T}},
$$

$V^{*}$ can be either positive or negative, depending upon whether $D$ decreases or increases with $p$, respectively. As can be seen from differentiating Eq. (4), when the pressure-dependence of $\lambda$, $\mathrm{v}$, and $f$ can be neglected, $V^{*}$ is the sum of two components,

$$
V^{*}=V^{f}+V^{m}
$$

where

$$
V f=\left(\frac{\partial G_{f}^{\mathrm{o}}}{\partial p}\right)_{\mathrm{T}}
$$

and

$$
V^{m}=\left(\frac{\partial G^{m}}{\partial p}\right)_{T}
$$

Neglecting variations in $v$ and $\lambda$ is practically always justified [6,7], but the pressure-dependence of $f$ may or may not be negligible, depending on whether there is a significant pressure-dependence to the 
interaction between the impurity and the defect ${ }^{1}$. The formation volume, $V f$, is the volume change in the system upon formation of a defect in its standard state; the migration volume, $V^{m}$, is the additional volume change when the defect reaches the saddle point in its migration path. $V f$ characterizes the pressure-dependence of the equilibrium point defect concentration (e.g., A-V complex for the vacancy mechanism; A on an interstitial site for the interstitialcy mechanism); $V^{m}$ characterizes the pressuredependence of the defect mobility. These volume changes are shown schematically in Fig. 1.

\section{FORMATION}

\section{VACANCY}

INTERSTITIALCY
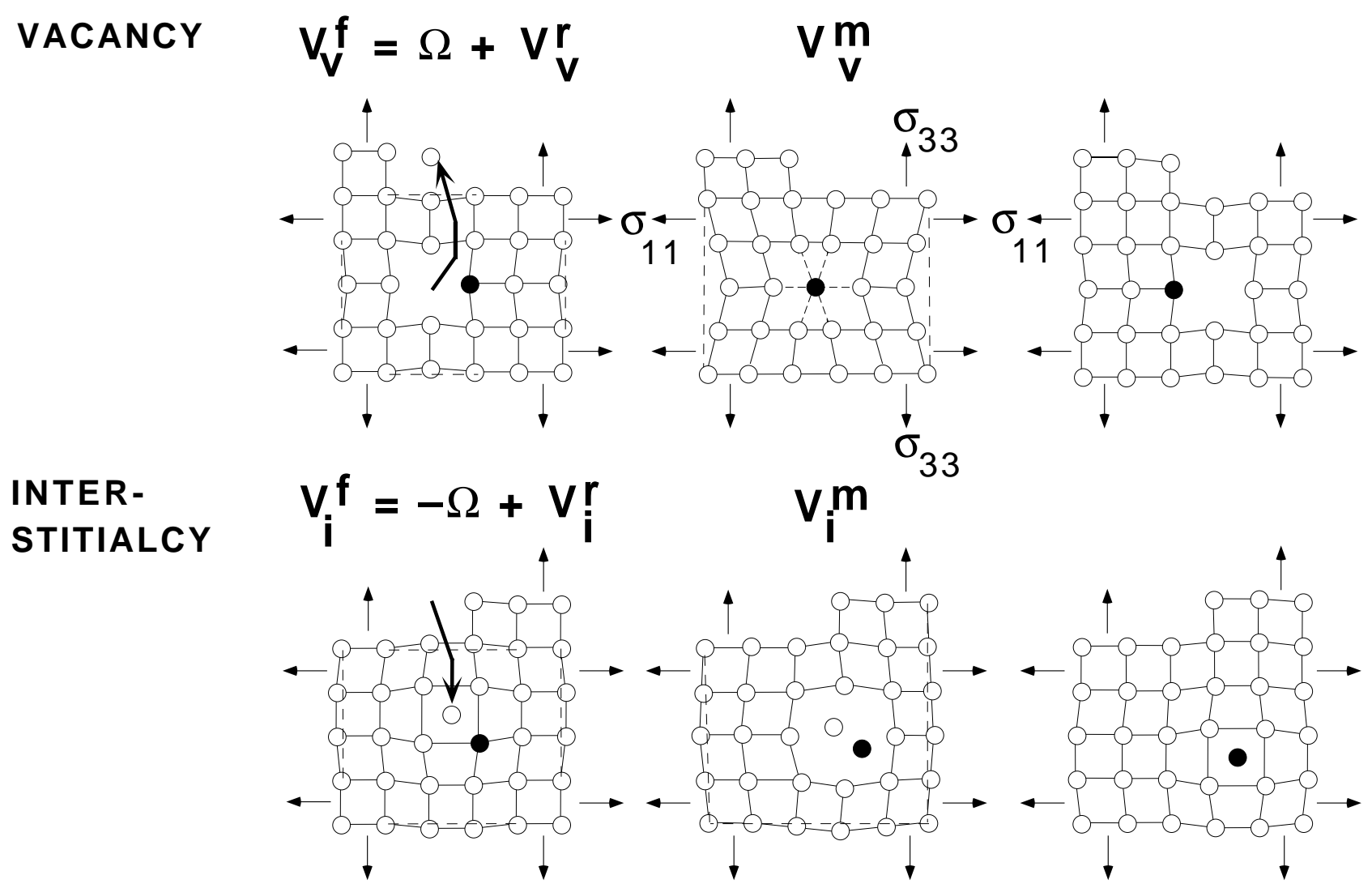

\section{MIGRATION}

Fig. 1. Schematic of volume changes associated with diffusion by vacancy (top) and interstitialcy (bottom) mechanism. Pressure effect is determined by volume changes of sample upon defect formation $\left(V^{f}\right)$ and migration $\left(V^{m}\right)$, which are shown. To create a vacancy, one moves an atom from the interior to a surface site; to create an interstitial, one moves an atom from a surface site to the interior. Stress effect is determined by shape change of sample upon defect formation and migration and, for formation, depends upon which surface the defect is formed

from. (If point defect equilibration occurs at an internal dislocation instead of the surface, dislocation climb results in an identical volume change; however, the shape change depends on

the direction of the Burgers vector.) Stress-strain work done by point defect formation and migration is indicated by interactions of shape changes with stresses (thin arrows).

A measurement of the activation volume can be a revealing test for distinguishing between potential diffusion mechanisms because, unlike activation energies, which are always positive, activation volumes are expected to have different signs for different types of point defect mechanisms. The formation volume is

\footnotetext{
${ }^{1}$ This pressure effect is analogous to the additional term in the expression for the apparent activation enthalpy introduced by a significantly temperature-dependent binding. The right-hand side of (6) and its temperature-equivalent expression gain an additional term, $-\mathrm{k}_{\mathrm{B}} \mathrm{T} \partial \ln f / \partial \mathrm{p}$ and $-\mathrm{k}_{\mathrm{B}} \partial \ln f / \partial(1 / \mathrm{T})$, respectively. The latter case has been analyzed by $\mathrm{Hu}$ [8].
} 


$$
V^{f}= \pm \Omega+V^{r}
$$

where $\Omega$ is the lattice site volume, and the plus sign is for vacancy formation, and the minus sign is for interstitial formation throughout this paper. The relaxation volume $V^{r}$ is the amount of outward relaxation of the sample surfaces (if the relaxation is inward, $V^{r}$ is negative) due to the newly-created point defect. $V^{r}$ is determined by, but is not necessarily equal to, the volume change of a shell of neighbors surrounding the point defect. If the shell is taken large enough that lattice distortions outside of it obey linear elasticity, then $V^{r}$ can be calculated directly from the expansion of the shell itself [9]. Because $V^{r}$ and $V^{m}$ are almost universally assumed to be much smaller than $\Omega$ in magnitude, the $\pm \Omega$ term is expected to dominate the pressure-dependence of $D$, and a positive (negative) activation volume is taken as indicative of a vacancy (interstitial) - based mechanism.

The advent of ab initio atomistic calculations of point defect energies and configurations permits the above assumptions to be lifted and a more rigorous test of point defect mechanisms to be performed using high pressure. Antonelli and Bernholc [10] calculated relaxation volumes for certain self diffusion pathways for neutral and positively-charged point defects. For the neutral vacancy mechanism they found $V_{V}^{r}=-0.24 \Omega$. The migration pathway for the interstitialcy mechanism [11] is still controversial, but for the tetrahedral interstitial ground state and bond-centered interstitial saddlepoint chosen by Antonelli and Bernholc, their results imply $V_{I-T e t}^{r}=+0.72 \Omega$ and $V_{I}^{m}=-0.28 \Omega$ for the neutral defect. For the direct interchange or "concerted exchange" mechanism (for which $V f \equiv 0$ because no point defect is involved), they found $V_{C E}^{m}=-0.04 \Omega$. Sugino and Oshiyama [12] calculated relaxation volumes for dopant diffusion via vacancy and interstitialcy mechanisms and the relaxation volume for vacancy formation in self-diffusion. For the latter their results imply $V_{V}^{r}=$ $-1.47 \Omega$. Other volumetric calculations have also been reported $[11,13,14]$. The disagreements among all of these calculations are not only due to differences among Hamiltonians (classical vs. Tight Binding Approximation vs. Local Density Approximation vs. Generalized Gradient Approximation). Significant differences also arise due to the implementation, e.g., the number of relaxational degrees of freedom that were permitted, the supercell size, and the method of deducing formation volumes from shell volume changes or from lattice parameter-dependencies of formation energies. In some cases the $\pm \Omega$ term in Eq. (9) has been overlooked. Nevertheless, it is apparent that the assumption of negligible relaxation and migration volumes should be made with great caution pending the outcome of more definitive atomistic calculations.

\section{EXPERIMENTS UNDER HYDROSTATIC PRESSURE}

There are very few measurements of the activation volume for diffusion in $\mathrm{Si}$. This is largely because such an experiment requires a pressure medium that neither reacts with, contaminates, nor plastically deforms silicon at high temperature. In early experiments we found negative activation volumes for $\mathrm{As} \mathrm{[15]} \mathrm{and} \mathrm{Si}$ [16] diffusion using a $\mathrm{NaCl}$ pressure medium. However, the $\mathrm{NaCl}$ pressure medium introduces substantial uncertainties into the results, especially for the self-diffusion study. Because $\mathrm{Si}$ is such a slow diffuser, this experiment had to be performed at $T$ above the $p=0$ melting point of $\mathrm{NaCl}$, requiring that the $p=0$ anneals be performed in a different furnace than the high- $p$ anneals. If the results hold up in planned "cleaner" experiments, the most obvious interpretation (making the assumptions discussed above) would be in terms of a pressure-induced increase in the interstitial population. More recently, activation volumes were measured for B and Ga diffusion in Si by Södervall et al. [17] using a gas apparatus. However, as the initial profiles were ion implanted, an undetermined amount of transient enhanced diffusion may be affecting the results.

We have now developed much cleaner, hydrostatic pressure environments for high-temperature annealing. We use a high-temperature Diamond Anvil Cell (DAC), cryogenically loaded with liquid argon, to provide a clean and hydrostatic pressure medium. The entire cell is externally heated by placing it into a furnace, which permits us to know the temperature to a high degree of accuracy and to guarantee its uniformity and reproducibility.

We had to develop techniques to measure the pressure quickly and reliably at high temperature in the DAC in order to perform these diffusion studies. Ordinarily for pressure measurement in a DAC one uses ruby fluorescence, the wavelength of which shifts with pressure. However, above around $200{ }^{\circ} \mathrm{C}$ the ruby signal becomes so faint and broad that it is virtually impossible to use. Fluorescence 
from Sm-doped YAG is still going strong as high as $850^{\circ} \mathrm{C}$, however, and we have now calibrated ten fluorescence peaks vs. $T$ and $p$ up to $850^{\circ} \mathrm{C}$ and $19 \mathrm{GPa}$ [18]. It turns out that we need all ten peaks in order to get a reliable pressure measurement at high $T$. Fitting ten fluorescence peaks which are fairly broad at high $T$ is not trivial, and therefore we will be distributing the necessary tools via the World-Wide Web so that others can readily use Sm:YAG for pressure measurement at high $T$.

With this new technique, we discovered pressure-enhanced interdiffusion of amorphous $\mathrm{Si} /$ amorphous Ge multilayers [19], and pressure-enhanced diffusion of As in crystalline Ge [20]. In the latter case the activation volume is very small in magnitude, in marked contrast to the relatively large positive activation volume found by Werner et al. [7] for Ge self-diffusion. The self-diffusion experiments support the "common wisdom" that diffusion in Ge occurs predominantly by a vacancy mechanism; however, the qualitatively different results for As diffusion do not appear consistent with a simple vacancy mechanism. Several potential explanations have been offered [21]. We are currently studying the diffusion of boron in $\mathrm{Si}$ with this new technique.

\section{DIFFUSION UNDER NONHYDROSTATIC STRESS}

The effect of biaxial strain on diffusion in semiconductor thin films is controversial at present, the reported strain effect varying from huge to barely discernible [22-28]. To understand the various influences of pressure and stress on the diffusivity [4,29], we consider separately the effects on the point defect concentrations [4,30-32] and on the point defect mobilities [33,34].

The thermodynamics of point defect formation in heavily dislocated crystals under hydrostatic or nonhydrostatic stress has been well understood for decades. When experimental conditions are such that the point defect concentrations equilibrate rapidly with the point defect sources and sinks compared to the experimental time scale, then the pressure-dependence of the point defect concentration is characterized by the (scalar) formation volume defined by Eq. (7). The new situation that gives the point defect formation volume in a cubic crystal a tensor character, is that large single crystals entirely free of extended defects, at which point defects can internally equilibrate, are now commonplace. Consequently, in a single crystal free of internal point defect sources, the volume change upon point defect formation does not tell the entire story: the crystal changes shape, the shape change depends upon which surface(s) the defects are equilibrated, and nonhydrostatic stresses interact with the components of that shape change in the characteristic stress-strain tensor manner. Below is a synopsis of the derivation [4] and of a more thorough explanation [29]. Let us first discuss the relevant effects for a bulk single crystal, and then discuss the case of a dislocation-free, coherently strained, epitaxial single crystal thin film.

Suppose that rapid heating of an initially perfect tile-shaped "wafer" from absolute zero results in the introduction of only monovacancies, that their relaxation volume is zero, and that the lattice parameter is independent of $T$ and $p$. Consider the stress-strain work done by the wafer on some agent of compressive stress applied to each set of faces in turn. A gravity-driven device for applying different stresses to each set of faces is shown in Fig. 2. Suppose that, when applying compressive stress to the lateral faces, we can do this without buckling the wafer (alternatively, we could use tensile stress). Assume that there are enough steps on the crystal surfaces that vacancies can be formed without changing the surface area. The fraction of vacant lattice sites in equilibrium (neglecting local entropy effects for the sake of simplicity) is a Boltzmann factor in the vacancy formation-induced change in the total energy of the system (energy of the crystal plus gravitational potential energy of the weights). An equivalent alternative statement is that the fraction of vacant lattice sites in equilibrium is a Boltzmann factor in the total energy change of the crystal itself, plus the work done against the agents imposing the stresses (in this case, gravity). If vacancies are formed at the (001) surfaces but not at the (100) surfaces (say, because the latter are coated with a film preventing them from serving as efficient sources/sinks), the work done against gravity is $p_{3} V f$; if vacancies are formed at the (100) surfaces but not at the (001) surfaces, then the work done against gravity is $p_{1} V f$. The equilibrium point defect concentrations depend on the location of the sources/sinks. A similar argument applies for the formation of self-interstitials. 


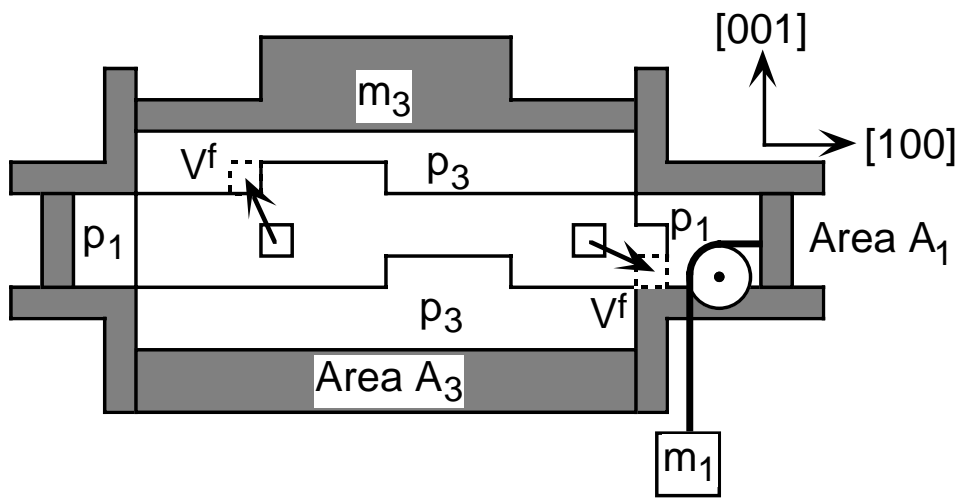

Fig. 2. Work done upon vacancy formation in crystal (middle) with stepped surfaces free of extended defects. Pressures, $p_{1}$ and $p_{3}$, of incompressible fluids in contact with (100) and (001) faces, respectively, maintained by pistons driven by masses $m_{1}$ and $m_{3}$. Crystal volume changes by $V f$ upon vacancy formation at (100) or (001) surface, but work done against gravity differs.

Now let us permit relaxation of the neighbors of the vacancy or self-interstitial. This relaxation propagates out isotropically to the surfaces of the sample, resulting in a volume change $V^{r}$. Unlike the volume change upon creation of unrelaxed point defects, the volume change upon relaxation interacts with the normal tractions applied to all surfaces. For point defects formed at the (001) surface, which is what we expect for diffusion measured near the free surface of any dislocation-free (001) wafer or thin film, the dimension changes of the crystal upon point defect formation can be described by the "formation strain tensor" $\mathbf{V}^{\mathbf{f}}$ :

$$
\mathbf{V}^{\mathbf{f}}= \pm \Omega\left[\begin{array}{lll}
0 & & \\
& 0 & \\
& & 1
\end{array}\right]+\frac{V^{r}}{3}\left[\begin{array}{lll}
1 & & \\
& 1 & \\
& & 1
\end{array}\right]
$$

The + sign is for vacancy formation, and the - sign is for interstitial formation throughout this paper. The dependence of the equilibrium vacancy concentration $C^{e}$ on applied stress $\sigma$ can be written

$$
\frac{C^{e}(\sigma)}{C^{e}(\mathbf{0})}=\exp \left(\frac{\sigma \cdot \mathbf{V}^{\mathbf{f}}}{k_{\mathrm{B}} T}\right)
$$

where $\mathbf{V}^{\mathbf{f}}$ has the elements 0 and 1 placed appropriately in Eq. (10) for the surface at which the defects equilibrate.

In general, the defect mobility, $M$, in directions parallel and perpendicular to the direction of applied stress, differ [33,34]. Typically, we measure $D_{33}$, the diffusivity in a direction normal to the surface, which depends on $M_{33}$, the mobility in this direction. The effect of stress on $M_{33}$ is characterized by the migration strain tensor, which must have the form

$$
\mathbf{V}_{\mathbf{3 3}}^{\mathbf{m}}=\left[\begin{array}{ccc}
V_{\perp}^{m} & & \\
& m & \\
& V_{\perp} & \\
& & V_{\|}^{m}
\end{array}\right] .
$$

$V_{\|}^{m}$ and $V_{\perp}^{m}$, respectively, are the dimension changes of the crystal parallel and perpendicular to the direction of net transport when the point defect reaches its saddle point. As the diffusivity is proportional to the product of the concentration and mobility of defects, the effect of stress on the diffusivity in the direction normal to the film surface is obtained by the combination of Eqs. (10) and (12):

$$
\begin{aligned}
& k_{\mathrm{B}} T \ln \frac{D_{33}(\sigma)}{D_{33}(\mathbf{0})}=\sigma \cdot \mathbf{V}_{\mathbf{3 3}}^{*} ; \\
& \mathbf{V}_{\mathbf{3 3}}^{*}=\mathbf{V}_{\mathbf{f}} \mathbf{V}_{\mathbf{3 3}}^{\mathbf{m}} .
\end{aligned}
$$


Hydrostatic pressure then influences $D_{33}$ according to

$$
\frac{D_{33}(p)}{D_{33}(0)}=\exp \left(\frac{-p\left[ \pm \Omega+V^{r}+V^{m}\right]}{k_{\mathrm{B}} T}\right)
$$

where the sum of the bracketed terms in Eq. (15) is the conventionally-defined scalar activation volume $V^{*}$, and the conventionally-defined scalar migration volume $V^{m}$ is the trace of Eq. (12). Biaxial stress influences $D_{33}$ according to

$$
\frac{D_{33}\left(\sigma_{\text {biax }}\right)}{D_{33}(0)}=\exp \left(\frac{\sigma_{\text {biax }}\left[\frac{2}{3} V^{r}+V^{m}-V_{\|}^{m}\right]}{k_{\mathrm{B}} T}\right) .
$$

Experimentally [22-28], the influence of biaxial stress has been characterized by the apparent change in activation energy with biaxial (tensile) strain, $\varepsilon$, at constant composition:

$$
Q^{\prime} \equiv-k_{\mathrm{B}} T \frac{\partial \ln D_{33}}{\partial \varepsilon}
$$

The controversy over the proper interpretation of the strain-dependence can now be addressed. By comparison to Eq. (16), the combination of volumes in square brackets in Eq. (16) is equal to $-Q^{\prime} / Y$, where the biaxial modulus $Y$ is the ratio of Young's modulus to one minus Poisson's ratio.

Additionally, one must take care to isolate experimentally the stress effect from the composition effect; this has been done in some of the most recent experiments [25,27,28].

For any proposed point defect mechanism, the three parameters $V^{r}, V_{\|}^{m}$, and $V_{\perp}^{m}$ can presently be calculated using molecular statics or dynamics simulations and the results used to predict the measured behavior through Eqs. (15) and (16), respectively. However, in principle it is possible to measure these parameters independently. Hydrostatic and biaxial stress experiments, through Eqs. (15) and (16) provide two equations in the three variables $V^{r}, V_{\|}^{m}$, and $V_{\perp}$. A third experiment is needed to uniquely identify these parameters from experimental measurements alone. One such experiment would be the effect of biaxial stress upon $D_{11}$, the diffusivity in a direction parallel to the film surface. An analysis of one proposed experiment [29] shows that the anisotropy in the migration strain itself, $V_{\|}^{m}-V_{\perp}$, can be found from its result:

$$
\frac{D_{11}\left(\sigma_{\text {biax }}\right)}{D_{33}\left(\sigma_{\text {biax }}\right)}=\exp \left(\frac{\sigma_{\text {biax }}\left[V_{\|}^{m}-V_{\perp}^{m}\right]}{k_{\mathrm{B}} T}\right) .
$$

\section{NONHYDROSTATIC STRESS: COMPARISON OF THEORY AND EXPERIMENT}

In the absence of atomistic calculations or the $D_{11}\left(\sigma_{\text {biax }}\right)$ experiment, we can examine and compare the calculations and experiments that have been performed to date. Combining Eqs. (12), (15), (16) and (17),

$$
V^{*}+\frac{3}{2} \frac{Q^{\prime}}{Y}= \pm \Omega+\left(\begin{array}{c}
m \\
V_{\|}
\end{array}-V_{\perp}^{m}\right)
$$

In interpreting atomistic calculations and experiments in the past, the assumption has been made almost universally that $V^{m}$ is negligible. If instead we make the less restrictive assumption that the anisotropy in $\mathbf{V}^{\mathbf{m}}$ is negligible, then the right-hand side of Eq. (23) should be $+1 \Omega$ for a vacancy 
mechanism and $-1 \Omega$ for an interstitial-based mechanism.

For the simple vacancy mechanism, or any mechanism whereby point defect jumps are in the $<111>$ directions, the anisotropy in the migration volume in $\{001\}$ films is identically zero because crystal symmetry dictates that the effect of, say, compression in the [001] and [100] directions on the rate of vacancy jumping in the [111] direction must be identical [4]. This is not necessarily the case for the interstitialcy or kick-out mechanism, in which the migration path may involve second-neighbor jumps in $\langle 110\rangle$ directions [11].

Eq. (23) was used [4] to compare the value of $Q^{\prime}$ reported by Kringhoj et al. [28] for Sb diffusion in biaxially strained $\mathrm{Si}-\mathrm{Ge}$ and $V^{*}$ calculated for the vacancy and interstitialcy mechanisms in hydrostatically compressed Si by Sugino and Oshiyama [12] using ab-initio methods. The result is $V^{*}+\frac{3}{2} \frac{Q^{\prime}}{Y}=(+0.93 \pm 0.20) \Omega$ for a compressively strained Sig1Ge9 alloy and $V^{*}+\frac{3}{2} \frac{Q^{\prime}}{Y}=(+1.20 \pm$ $0.33) \Omega$ for tensile Si. With no free parameters, these results are in excellent agreement with the prediction of $+1 \Omega$ for a simple vacancy mechanism. Graphically, this agreement is illustrated in Fig. 3.

A similar analysis has been performed for the existing experiments on B diffusion in $\mathrm{Si}$ [29], yielding qualitative agreement with a predominantly interstitial-based mechanism. However, in this case there are several unknown parameters the determination of which, via calculations and/or experiments, would be very valuable. The missing parameters are the formation or relaxation volumes for B diffusion by the interstitialcy or kick-out mechanism in the ground state and at the saddle point of the migration path, and the anisotropy in the migration strain.

\section{CALCULATING ACTIVATION STRAINS}

If one has an atomistic mechanism in mind for diffusion, the energy and volume changes for the various elementary steps may be calculated using molecular statics or dynamics, and the result compared to measured temperature and pressure-dependencies of the diffusivity. In general, the full tensor character of the volume change (the activation strain) can be calculated and compared with experimental results obtained under nonhydrostatic stress, permitting a more exacting test of the proposed mechanism. In principle, the simplest way to calculate the activation strain is identify the nearest-neighboring atoms to the location where the configuration change is occurring, and to calculate their displacements out to a distance where nonlinear elastic effects become negligible. Continuum elasticity then permits the evaluation of the displacements of the sample surfaces, which directly gives the activation strain. In practice, it has been simplest to calculate the dependence of the energy changes upon the imposed lattice parameter; deducing volume changes in this case requires making assumptions about the entropy changes[10]. A more direct practical method that is now becoming possible is to permit all three lattice parameters in a supercell to vary during atomistic configuration changes occurring at zero applied stress; variations in the lattice parameters then give the activation strain tensor directly. 


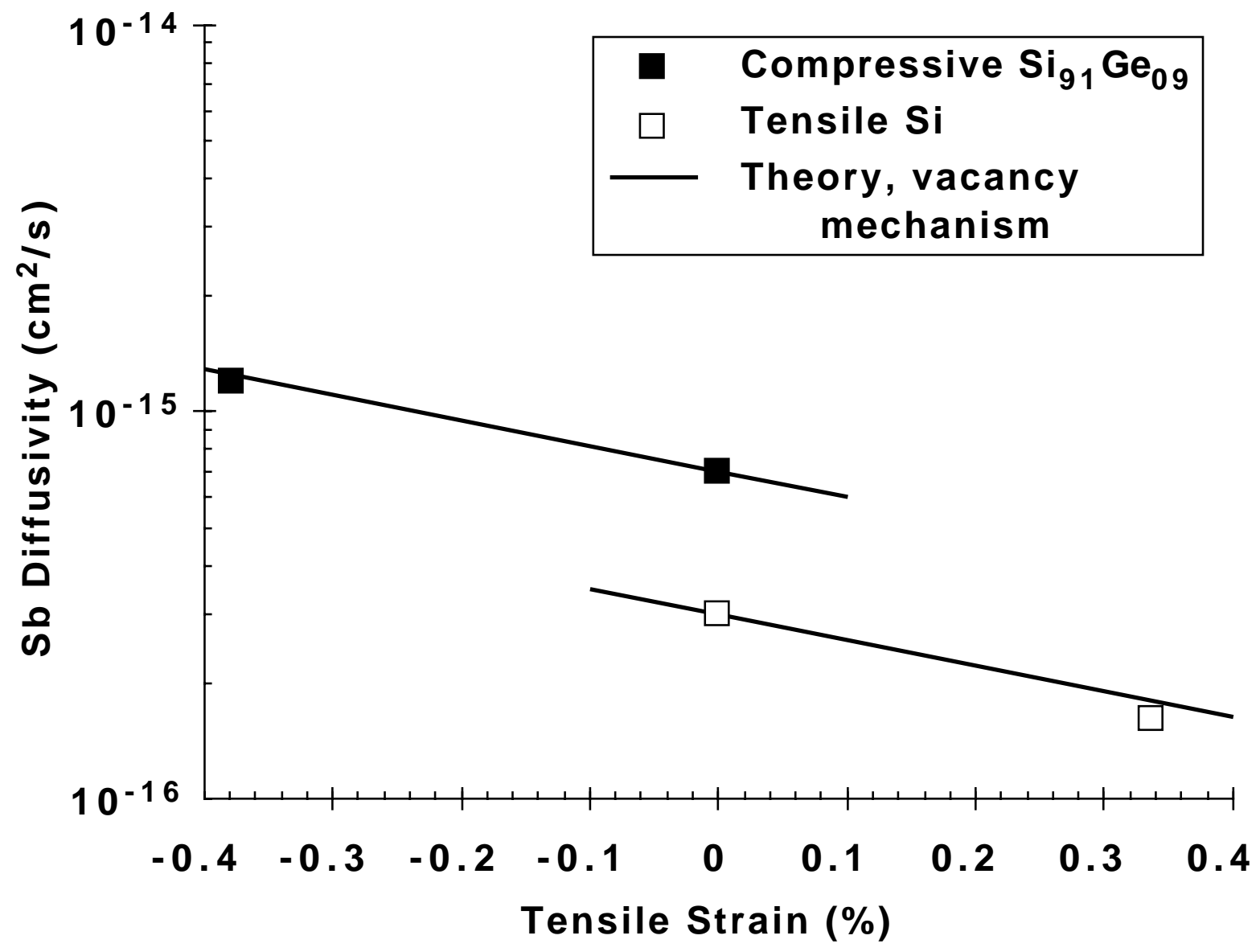

Fig. 3. Biaxial strain effect on Sb diffusion in Si-Ge thin film at constant composition. Data points represent average values from Kringhoj et al. over range 900-1030 ${ }^{\circ} \mathrm{C}$. The slopes of the lines indicate the value of $Q^{\prime}$ expected from the biaxial experiment by inputting Sugino and Oshiyama's result for the vacancy mechanism under hydrostatic conditions into Eq. (23) with no free parameters. The offset between lines is a free parameter representing the effect of composition at constant strain; lines have been chosen to go through the data points at zero strain.

\section{SUMMARY}

The effects of hydrostatic pressure and nonhydrostatic stress on diffusion are related to each other and to calculable atomistic parameters through the thermodynamic equations (13) and (23). There is quantitative agreement between the calculated atomistic parameters for the vacancy mechanism and the biaxial strain experiment for the case of $\mathrm{Sb}$ diffusion, with no free parameters. There is qualitative agreement for an interstitial-based mechanism for the case of B diffusion; however, values for key parameters are missing. Critical experiments and calculations for the determination of these key parameters are pointed out.

\section{ACKNOWLEDGMENTS}

This research was supported by NSF-DMR-95-25907.

\section{REFERENCES}

${ }^{1}$ P.M. Fahey, P.B. Griffin and J.D. Plummer, Rev. Mod. Phys. 61 (1989), p. 289.

${ }^{2}$ P.M. Fahey, G. Barbuscia, M. Moslehi and R.W. Dutton, Appl. Phys. Lett. 46 (1985), p. 784.

${ }^{3}$ Y. Zhao, M.J. Aziz, S. Mitha and D. Schiferl, Mater. Res. Soc. Symp. Proc. 442 (1997), p. 305. 
${ }^{4}$ M.J. Aziz, Appl. Phys. Lett. 70 (1997), p. 2810.

${ }^{5}$ G.H. Vineyard, J. Phys. Chem. Solids 3 (1957), p. 121.

${ }^{6}$ R.N. Jeffery and D. Lazarus, J. Appl. Phys. 41 (1970), p. 3186.

${ }^{7}$ M. Werner, H. Mehrer and H.D. Hochheimer, Phys. Rev. B 31 (1985), p. 3930.

${ }^{8}$ S.M. Hu, Phys. Stat. Sol. (b) 60 (1973), p. 595.

9.D. Eshelby, Solid State Physics 3 (1956), p. 79.

${ }^{10}$ A. Antonelli and J. Bernholc, Phys. Rev. B 40 (1989), p. 10643.

${ }^{11}$ M. Tang, L. Colombo, J. Zhu and T. Diaz de la Rubia, Phys. Rev. B 55 (1997), p. 14279.

${ }^{12}$ O. Sugino and A. Oshiyama, Phys. Rev. B 42 (1990), p. 11869.

${ }^{13}$ H. Seong and L.J. Lewis, Phys. Rev. B 53 (1996), p. 9791.

${ }^{14}$ M. Nastar, V.V. Bulatov and S. Yip, Phys. Rev. B 53 (1996), p. 13521.

${ }^{15}$ E. Nygren, M.J. Aziz, D. Turnbull, J.M. Poate, D.C. Jacobson and R. Hull, Appl. Phys. Lett. 47 (1985), p. 105.

${ }^{16}$ M.J. Aziz, E. Nygren, W.H. Christie, C.W. White and D. Turnbull, Mater. Res. Soc. Symp. Proc. 36 (1985), p. 101.

${ }^{17}$ U. Södervall, M. Friesel and A. Lodding, J. Chem. Soc. Faraday Trans. 86 (1990), p. 1293.

${ }^{18}$ Y.C. Zhao, W. Barvosa-Carter, S.D. Theiss, S. Mitha, M.J. Aziz and D. Schiferl (submitted).

${ }^{19}$ S.D. Theiss, F. Spaepen and M.J. Aziz, Appl. Phys. Lett. 68 (1996), p. 1226.

${ }^{20}$ S. Mitha, M.J. Aziz, D. Schiferl and D.B. Poker, Appl. Phys. Lett. 69 (1996), p. 922.

${ }^{21}$ S. Mitha, M.J. Aziz, D. Schiferl and D.B. Poker, Defect and Diffusion Forum 143-147 (1997), p. 1041.

${ }^{22}$ N. Moriya, L.C. Feldman, H.S. Luftman, C.A. King, J. Bevk and B. Freer, Phys. Rev. Lett. 71 (1993), p. 883.

${ }^{23}$ F.H. Baumann, J.-H. Huang, J.A. Rentschler, T.Y. Chang and A. Ourmazd, Phys. Rev. Lett. 73 (1994), p. 448.

${ }^{24}$ N.E.B. Cowern, P.C. Zalm, P. van der Sluis, D.J. Gravesteijn and W.B. de Boer, Phys. Rev. Lett. 72 (1994), p. 2585.

${ }^{25}$ N.E.B. Cowern, W.J. Kersten, R.C.M. de Kruif, J.G.M. van Berkum, W.B. de Boer, D.J. Gravesteijn and C.W.T. Bulle-Liewma, in Proc. 4th Int. Symp. on Process Physics and Modeling in

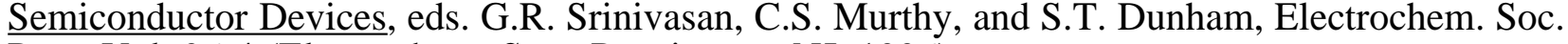
Proc. Vol. 96-4 (Electrochem. Soc., Pennington, NJ, 1996).

${ }^{26}$ P. Kuo, J.L. Hoyt, J.F. Gibbons, J.E. Turner, R.D. Jacowitz and T.I. Kamins, Appl. Phys. Lett. 62 (1993), p. 612.

${ }^{27}$ P. Kuo, J.L. Hoyt, J.F. Gibbons, J.E. Turner and D. Lefforge, Appl. Phys. Lett. 66 (1995), p. 580.

${ }^{28}$ P. Kringhoj, A. Nylandsted-Larsen and S.Y. Shirayev, Phys. Rev. Lett. 76 (1996), p. 3372.

${ }^{29}$ M.J. Aziz, Mater. Res. Soc. Symp. Proc. 469 (1997), in press.

${ }^{30}$ F.C. Larché and J.W. Cahn, Acta Metall. 33 (1985), p. 331.

${ }^{31}$ B.J. Spencer, P.W. Voorhees and S.H. Davis, J. Appl. Phys. 73 (1993), p. 4955.

${ }^{32}$ H. Park, K.S. Jones, J.A. Slinkman and M.E. Law, J. Appl. Phys. 78 (1995), p. 3664.

${ }^{33}$ P.H. Dederichs and K. Schroeder, Phys. Rev. B 17 (1978), p. 2524.

${ }^{34}$ M.J. Aziz, P.C. Sabin and G.-Q. Lu, Phys. Rev. B 44 (1991), p. 9812.

*maziz@harvard.edu; http://www.deas.harvard.edu/matsci 